\title{
Propulsion Controls Modeling for a Small Turbofan Engine
}

\author{
Joseph W. Connolly* \\ NASA Glenn Research Center, \\ Cleveland, $\mathrm{OH}$ 44135, USA
}

\author{
Jeffrey T. Csank ${ }^{\dagger}$ \\ NASA Glenn Research Center, \\ Cleveland, $\mathrm{OH}$ 44135, USA
}

Amy Chicatelli

Vantage Partners LLC, Brook Park, OH 44142, USA

Kevin Franco ${ }^{\S}$

University of California - Riverside, Riverside, CA 92521, USA

\begin{abstract}
A nonlinear dynamic model and propulsion controller are developed for a small-scale turbofan engine. The small-scale turbofan engine is based on the Price Induction company's DGEN 380, one of the few turbofan engines targeted for the personal light jet category. Comparisons of the nonlinear dynamic turbofan engine model to actual DGEN 380 engine test data and a Price Induction simulation are provided. During engine transients, the nonlinear model typically agrees within $10 \%$ error, even though the nonlinear model was developed from limited available engine data. A gain scheduled proportional integral low speed shaft controller with limiter safety logic is created to replicate the baseline DGEN 380 controller. The new controller provides desired gain and phase margins and is verified to meet Federal Aviation Administration transient propulsion system requirements. In understanding benefits, there is a need to move beyond simulation for the demonstration of advanced control architectures and technologies by using real-time systems and hardware. The small-scale DGEN 380 provides a cost effective means to accomplish advanced controls testing on a relevant turbofan engine platform.
\end{abstract}

\section{Nomenclature}

$\begin{array}{llll}A & \text { State system matrix } & P R & \text { Pressure Ratio } \\ B & \text { Input system matrix } & P s 3 & \text { High pressure compressor static discharge pressure } \\ C & \text { Output system matrix } & T 4 & \text { Combustor exit temperature } \\ D & \text { Feed through system matrix } & W_{f} & \text { Fuel flow } \\ I & \text { Inertia } & s & \text { Denotes scalar quantity } \\ J & \text { Jacobian } & u & \text { Actuator command vector } \\ N_{c} & \text { Corrected speed (either spool) } & x & \text { State vector } \\ F_{N} & \text { Engine net thrust } & y & \text { Measured outputs vector } \\ W_{c} & \text { Corrected flow } & \eta & \text { Efficiency } \\ W_{f} & \text { Fuel flow, lb/s or } \% \text { full value } & k & \text { Operating point index }\end{array}$

$N_{H} \quad$ High pressure shaft rotational speed, rpm

$N_{L} \quad$ Low pressure shaft rotational speed, rpm

\footnotetext{
*Aerospace Engineer, Intelligent Controls and Autonomy Branch, 21000 Brookpark Rd., 77-1, AIAA Senior Member

$\dagger$ Aerospace Engineer, Intelligent Controls and Autonomy Branch, 21000 Brookpark Rd., 77-1, AIAA Senior Member

¥Aerospace Engineer, 3000 Aerospace Parkway VPL-3, AIAA Senior Member

$\S$ Mechanical Engineer, 900 University Ave., Student
} 


\section{Introduction}

Any advanced control algorithms have been applied to commercial turbofan engines; however, these $\mathrm{I}_{\text {studies have largely been conducted in a simulation-only environment. They are "paper" models that }}$ cannot be adequately tested for real-world conditions. ${ }^{1-9}$ To alleviate this issue, NASA has recently acquired the Price Induction DGEN 380 engine and built around it the DGEN Aero-propulsion Research Turbofan (DART) facility. This facility enables aero-propulsion technology studies at the system level to be examined on a relevant platform and move beyond the typical simulation system studies. The work presented here provides a baseline control architecture applied to a nonlinear dynamic simulation representative of the DGEN 380 engine. This baseline nonlinear dynamic model will enable comparison testing of advanced controls in the DART facility to improve upon the technology readiness level of previous simulation studies. ${ }^{10-12}$

The DGEN family of engines are small, dual spool, high-bypass, geared turbofan engines used in several system level technology studies. ${ }^{13-16}$ A cut out side view of the DGEN 380 engine is shown in Fig. $1 .{ }^{17}$ The small-scale nature of the engine provides a means for hardware testing of a turbofan engine at a modest cost. Prior to the engine purchase, and the building of the DART facility at NASA, an engine test was performed to acquire data and enable a preliminary noise study. ${ }^{18,19}$

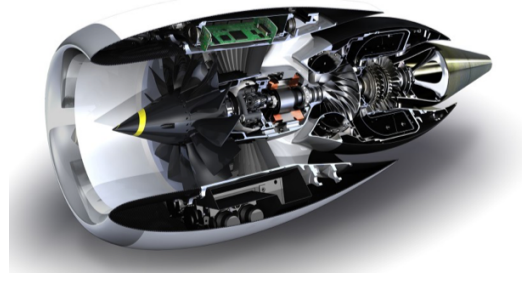

Figure 1: The Price Induction DGEN 380 small scale turbofan engine. ${ }^{20}$

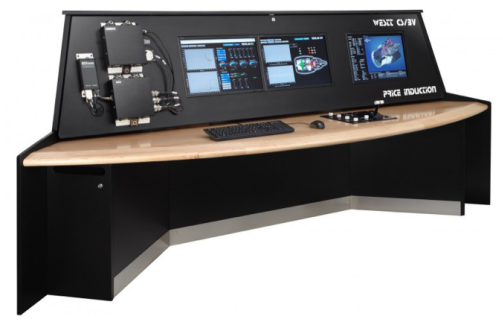

Figure 2: The Price Induction WESTT CS/BV Virtual Engine Test Bench. ${ }^{13}$

This initial engine test and the subsequent DART facility can only accommodate ground testing. To overcome this restriction and explore the full flight envelope of the DGEN 380, the WESTT CS/BV virtual engine test bench, also developed by Price Induction, is used for comparisons. The WESTT test bench is a multipurpose test bench modeled after the DGEN 380 engine system, employing a linear parameter varying (LPV) simulation including the engine control unit. The WESTT CS/BV simulation test bench, shown in Fig. 2, is targeted for practical education and research. The LPV model can improve accuracy across the flight envelope over a linear point model; however, for advanced engine controls to be implemented, a nonlinear physics-based engine model is desired. This type of model can capture the primary dynamic response across all operating conditions, and provide insight for off design conditions. The motivation for the work presented in this paper is to develop a suitable nonlinear engine model that can support advanced control studies.

To enable controls design, even while lacking important engine data such as component performance maps, a nonlinear model is developed using the Toolbox for the Modeling and Analysis of Thermodynamic Systems (T-MATS). ${ }^{21}$ The development of the engine model, prior to having all of the desired engine data, provides a means for control algorithm development prior to the completion of the new DART facility. A baseline gain scheduled proportional integral low pressure shaft speed controller is developed with limiter safety logic. The controller provides desired gain and phase margins and is tested to meet Federal Aviation Administration transient propulsion system requirements. This baseline controller will serve as a comparison algorithm to future advanced control studies.

This paper provides an overview of the DGEN 380 engine and discusses a method for developing the DGEN 380 engine nonlinear model from limited available engine data. The nonlinear model is then used for the development of a baseline controller with limit logic to ensure safe operation. Results are presented for modeling accuracy regarding stability margins of the controller and the controlled dynamic response of the engine relative to actual engine test data and the simulation from the manufacturer's WESTT test bench. Finally, conclusion of the propulsion controls development for the small scale DGEN 380 engine are presented. 


\section{DGEN 380 Engine Overview}

The DGEN family of engines are small, dual spool, high-bypass, geared turbofan engines manufactured by Price Induction. These engines are intended for personal light jets, which are defined as 4 to 6 seat aircraft with a maximum take-off weight between $3,638 \mathrm{lb}(1650 \mathrm{~kg})$ to $4,740 \mathrm{lb}(2150 \mathrm{~kg})$. The notional aircraft operates at altitudes around 10,000 ft with a maximum ceiling of 25,000 ft and at speeds less than 250 ktas. This section will provide information on the DGEN 380 engine and the Price Induction WESTT test bench simulation.

\section{A. Engine Hardware}

The DGEN 380 engine contains four primary turbo-machinery components. The high pressure turbine (HPT) connects to the high pressure compressor (HPC) via the high speed shaft (HS). The low pressure turbine (LPT) is connected to the low speed shaft (LS) and drives the fan via speed reducer gear box. In this model, the fan is modeled as two individual components. The hub (FanHub), which is a booster to the core flow, and the tip (FanTip) which provides the bypass flow that goes around the core of the engine. Figure 3 contains the component designations and station numbers used in the model. The DGEN 380 has a bypass ratio of 7.6 and features a geared turbofan with a 3.32 ratio that links the low pressure spool to the fan spool. It operates at a cruise operating point of approximately 10,000 ft and $0.3 \mathrm{Mach}$.

Additional features of the turbofan engine of interest to controls model developer are the available sensors, fuel flow actuator, bleed flow, and cooling flow. The sensors used in this study are the LS or low pressure shaft speed $\left(N_{L}\right)$, the HS or high pressure shaft speed $\left(N_{H}\right)$, the compressor static pressure (Ps3), the fuel flow $\left(W_{f}\right)$, and exhaust gas temperature $(E G T$, or T5). The fuel flow actuator is an electric design with a bandwidth of approximately $6 \mathrm{~Hz}$. The bleed and cooling flow are scheduled based on operating condition and are not actively controlled.

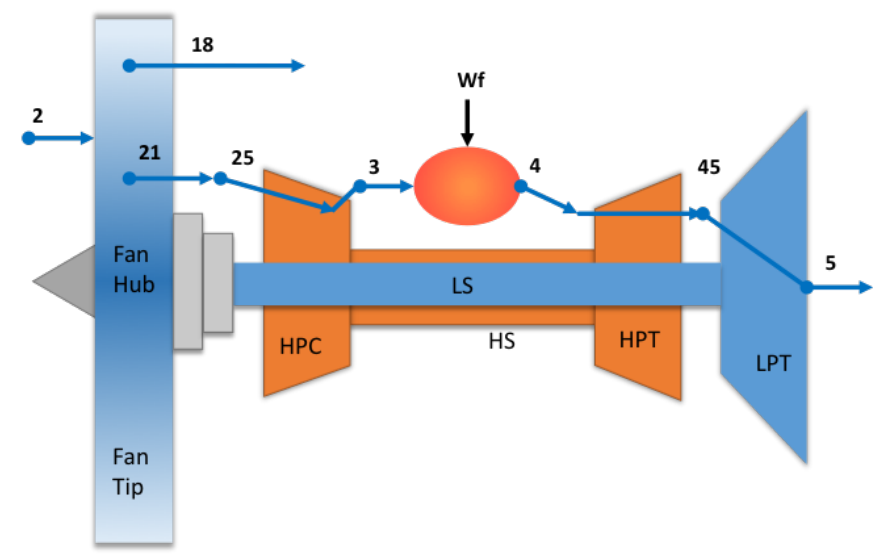

Figure 3: DGEN 380 engine schematic with main turbomachinery components.

The DART facility is housed within the Aero-Acoustic Propulsion Laboratory (AAPL) dome, ${ }^{22}$ a ground engine test facility that is suitable for acoustic studies and future advanced control studies. The dome is $65 \mathrm{ft}$ high and $130 \mathrm{ft}$ in diameter, providing an anechoic testing environment for engine component research and development. The DGEN380 engine is mounted on a mobile engine test stand. Acoustic wedges were placed on the bed and back cab of the truck to minimize reflections. Figure 4 shows the DGEN 380 and thrust stand in the AAPL. Data were acquired with the DGEN 380 engine operating over a $N_{L}$ range of flight idle to full power. 


\section{B. Price Induction Simulation - WESTT Test Bench}

The Price Induction WESTT test bench is used to enable the development of a propulsion controller beyond the ground test data to a full flight envelope. This is a simulation for the study of the DGEN 380 turbofan and its control, shown in Fig. 2. ${ }^{13}$ The test bench uses flight-like controller hardware and an LPV engine model running in real-time. The WESTT features a real time simulation of the engine performance data, dynamic $3 \mathrm{D}$ visualization of the engine, thermodynamic and aerodynamic graphical explorations, and the DGEN control system with access to the engine regulation code. The test bench is also being used to integrate with NASA-developed distributed engine control technologies. ${ }^{23}$ Data was obtained from the WESTT simulation at several cruise operating points for comparisons to the developed nonlinear engine model. The WESTT test bench provides simulation output for all of the available sensors on the DGEN 380 engine with the addition of pressures, temperatures, and mass flow rates at all engine component locations. Without the use of component performance maps the WESTT test bench does not provide operability margins such as HPC stall margin.

\section{DGEN 380 Nonlinear Model}

A nonlinear dynamic model of the DGEN 380 engine is required for the development of advanced controls algorithms that can then be tested in the DART facility. This type of model enables controls testing in off design operation, the study of engine operability margins, and confidence for the controller design prior to testing on hardware. The model developed for this study is using the Toolbox for the Modeling and Analysis of Thermodynamic Systems (T-MATS). ${ }^{21}$ T-MATS is an open-source toolbox written and developed for use in MATLAB/Simulink. T-MATS contains generic thermodynamic components and basic control functions that can be combined with a variable input iterative solver and optimization algorithm to create complex physics-based systems. One feature of T-MATS is the turbo-machinery block set, which provides the developer tools required to create steady-state or dynamic turbo-machinery simulations. This section will outline the development of the nonlinear dynamic DGEN 380 model and specific challenges in developing such a model with limited data.

\section{A. Toolbox for the Modeling and Analysis of Thermodynamic Systems}

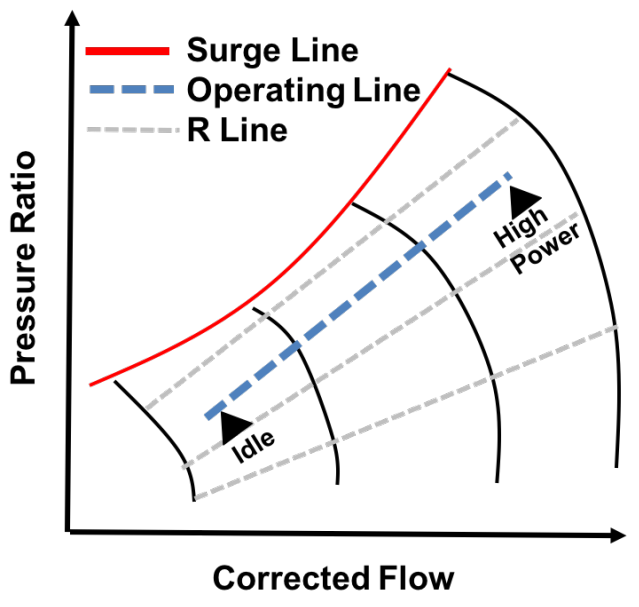

Figure 5: Generic fan map showing the operationalline (dashed blue), surge (red), speed lines (black) and R-lines (dashed grey).

of performance map data was the primary limitation of available data, and the scaling of generic maps is discussed in greater detail in the subsequent subsection. MATS library are used for the fan hub, fan tip and HPC components, while generic T-MATS turbine blocks are used for the HPT and LPT. The individual turbo-machinery components are assumed to be 0-dimensional, where the component-level gas dynamics are not modeled. Internal component calculations contain a combination of physics based and empirical models. Algebraic relations and component maps are used to generate component or system steady state solutions at specific operating points. For example, compressor efficiency $(\eta)$, corrected mass flow $\left(W_{R}\right)$, and pressure ratio $(P R)$ are determined from the compressor map as functions of corrected shaft speed $\left(N_{c}\right)$ and R-line, where R-line is a uniquely defined line on the compressor map. A generic illustration of a compressive component performance map is shown in Fig. 5. Component maps for the DGEN 380 engine are unavailable and not part of the WESTT test bench. Consequently, a generic set of component maps are used for all the compressor and turbine components. The lack

For this model, the primary components are shown in Fig. 3. Generic compressor blocks from the T- 
The individual turbo-machinery components are connected such that the flow from the inlet (ambient conditions) is connected to a splitter block that splits the flow into two paths. The bypass flow path connects the branch flow output to the fan tip, while the core flow path is connected to the fan hub. Both fan components (FanTip and FanHub) are connected to the LS through the T-MATS Gear Box block. The gear box and LPT torques are summed to comprise the input to the LS. The HPC, HPT, and a generator torque are summed to comprise the input torque to the HS, which computes the rotor accelerations. In addition, a generator T-MATS block is included on the HS to represent the electrical component of the DGEN 380 engine. The input to the generator block is the power demand (or source) at full power and the HS speed. The power demand is approximately several kilo-Watts at full speed. The unbalanced torques on the shafts provide the dominant dynamic response of the turbofan engine, where the shaft accelerations $\left(\dot{N}_{n}\right)$ are calculated as shown in Eq. 1.

$$
\dot{N}_{n}=\frac{\sum\left(\tau_{i}\right)}{2 \pi I}
$$

To reach a balanced solution for the turbofan engine, T-MATS solvers are based around an iterative solver that operates with information defined by a Jacobian calculator shown in Fig. 6 . The rotor accelerations and the flow errors from each component, including the nozzle, are inputs to the Jacobian calculator block. The Jacobian calculator determines the independent variables; Rline for compressors, pressure ratio for turbines, total flow for inlet, splitter ratio, and updated rotor speeds. The iterative solver makes use of the NewtonRaphson (NR) method to step a plant toward a solution, a process described mathematically in Eq. 2, where $k$ is the step iteration number.

$$
x(k+1)=x(k)-\frac{f(x(k))}{J}
$$

The Jacobian $(J)$ is a linear map between the inputs $(x)$ and outputs $(f(x))$ of a plant and is defined by perturbing each $x$ from its initial conditions $(x(0))$ to find the effect on $f(x)$. A mathematical description of the Jacobian is given in Eq. 3. For non-linear systems, the Jacobian is only valid in a neighborhood local to the linearization point.

$$
J=\left[\begin{array}{ccc}
\frac{\partial f_{1}}{\partial x_{1}} & \cdots & \frac{\partial f_{1}}{\partial x_{n}} \\
\vdots & \ddots & \vdots \\
\frac{\partial f_{m}}{\partial x_{1}} & \cdots & \frac{\partial f_{m}}{\partial x_{n}}
\end{array}\right]
$$

These two equations, Eq. 2 and Eq. 3, represent the two main steps of operation in a T-MATS solver. Initially, the Jacobian calculator component creates a linear representation of the plant at the current operating point. This requires perturbing slightly, in turn, each input from its initial condition, recording the results, and numerically determining the partial derivative of each output with respect to each input. The Jacobian is built from these calculations, inverted, and provided to a NR solver. In the second step, the NR solver steps toward a solution using the Jacobian developed in the first step. In T-MATS, the method described above is used as the basis for a steady-state and a dynamic solver. Once the steady-state model has been developed, the next step is to add in the shaft dynamics (inertias). The simulation is modified to add additional layers to the model as shown in Fig. 6. The innermost layer is the component model level, which is where the component level model is connected to the solver. The independent values (Rlines, pressure ratios, total air flow, and splitter ratio) are obtained through the solver by driving component flow errors to zero.

\section{B. Modeling with Limited Engine Data}

To reduce controller development time while waiting for engine characterization testing in the DART facility, generic performance maps are used to initiate the nonlinear engine model development in T-MATS. These component maps need to be correctly scaled/sized to fit the available engine data. In this case, the engine data that the maps are scaled to come from the WESTT test bench simulation. For each component map, 


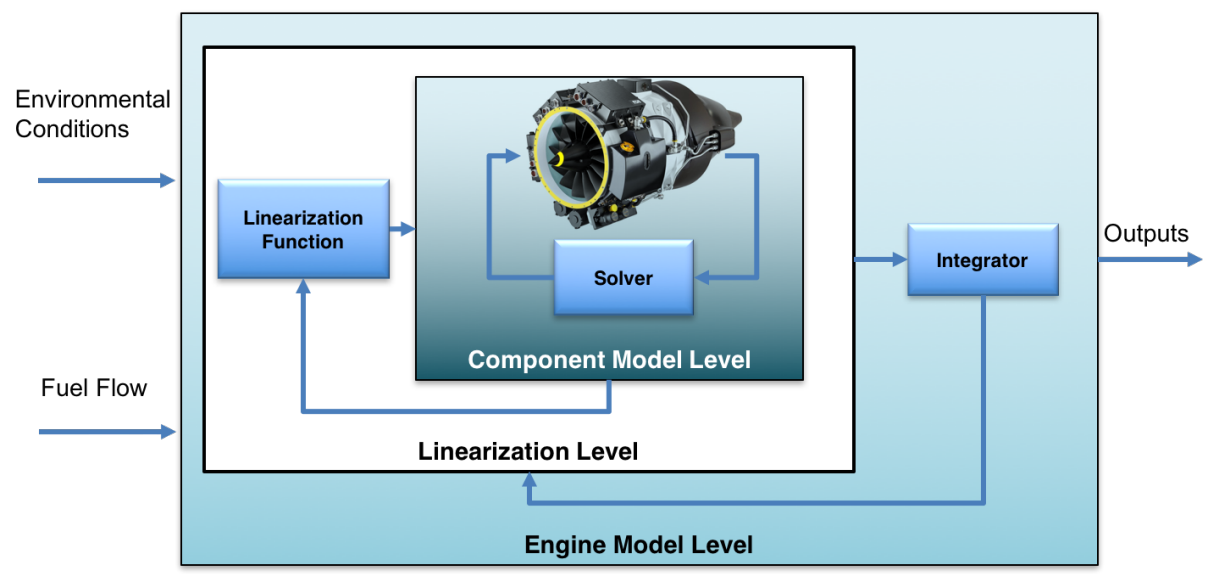

Figure 6: Diagram showing the various solver layers of the nonlinear engine model.

including both compressor and turbine types, there are four scalars calculated: flow $\left(s W_{c}\right)$, pressure ratio $(s P R)$, efficiency $(s \eta)$, and rotor speed $\left(s N_{c}\right)$. The scalars can be calculated manually using the following equations:

$$
\begin{array}{r}
s W_{c}=\frac{W_{c}}{W_{c, \text { map }}} \\
s P R=\frac{P R-1}{P R_{\text {map }}-1} \\
s \eta=\frac{\eta}{\eta_{\text {map }}} \\
s N_{c}=\frac{N_{c}}{N_{c, \text { map }}}
\end{array}
$$

where the actual corrected air flow rate $\left(W_{c}\right)$, pressure ratio $(P R)$, efficiency $(\eta)$ and corrected speeds $\left(N_{c}\right)$ are divided by the corresponding point on the component map $\left(W_{c, \text { map }}, P R_{\text {map }}, \eta_{\text {map }}\right.$, and $\left.N_{c, \text { map }}\right)$. In addition to these components, a nozzle for both the core (attached to the output of the LPT) and bypass flows (attached to the exit of the fan tip) are included. These inclusions require that the user provide the exit area of each nozzle.

To tune the shaft inertias, a transient fuel flow signal is recorded from the WESTT CS/BV test bench during which its engine model is operated at various flight conditions. The conditions of most interest are takeoff and cruise. The fuel flow input is then applied to the T-MATS DGEN engine model and the resulting shaft speeds are compared to the corresponding speeds obtained from the WESTT test bench. Subsequently, the inertia values for each shaft are modified until a satisfactory match has been found.

\section{Controller Development}

A baseline control architecture for a turbofan engine equipped with a full authority digital engine control is shown in Fig. 7. Turbofan engine controllers are designed to regulate a measurable variable such as low pressure shaft speed $\left(N_{L}\right)$, which is correlated with thrust. ${ }^{24}$ This is done since there is no on-board sensor for thrust. The main interface between the pilot and the control system is the power lever angle (PLA) or throttle, which is used by the controller logic to set a required $N_{L}$ command. The measured $N_{L}$ is subtracted from the command set point to create an error signal that goes to the controller to determine a fuel flow rate $\left(W_{f}\right)$. Protection logic limits the commanded fuel flow rate to provide safe operation of the engine. ${ }^{25-29}$ Finally, the fuel flow signal is sent to the fuel metering valve. The feedback controller and limiters are designed as gain scheduled proportional-integral (PI) control with integrator windup protection. The controller development for the nonlinear T-MATS model is provided in the following subsections, starting with the linearization of the nonlinear model to use classical control theory approaches, followed by an 
overview of the main fuel flow control, and then protection logic limiters.

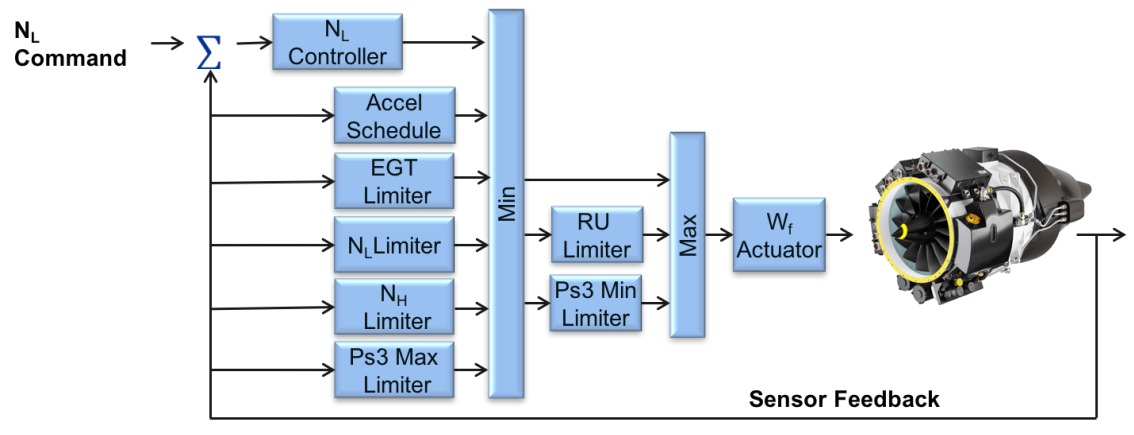

Figure 7: Baseline full authority digital controller diagram with a min/max protection logic.

\section{A. Piece-Wise Linear Model}

The aircraft turbofan engine has to operate over a wide range of environmental conditions. The fact that the engine behaves differently at different environmental conditions adds to the complexity of the control design. One way of handling this complexity is to use gain scheduling, ${ }^{30-32}$ which takes advantage of interpolation in order to transition between conditions. To develop this type of a controller the nonlinear model needs to be linearized across the various operating conditions. This is typically done using either a piece-wise linear model (PWLM) or an LPV model. The PWLM approach is used for this study. T-MATS handles the linearization of the nonlinear model through the calculated Jacobian included in the engine model shown in Fig. 6 . This linearization block is used to develop linear models at user-specified operating points. Furthermore, a PWLM of the engine is developed by executing the model at varying flight conditions and fuel flow inputs. The linearization block perturbs the inputs and states of the model to develop the state space linear model of the plant shown in Eq. 8.

$$
\begin{aligned}
& \dot{x}=A_{k} x+B_{k} u \\
& y=C_{k} x+D_{k} u
\end{aligned}
$$

The PWLM for this study spans six altitude points from sea level to $10,000 \mathrm{ft}$, seven equally spaced Mach number points from 0 to 0.33 , and eight $N_{L}$ speeds from flight idle to maximum power culminating in 336 distinct linear state space models. These models are then linked together to approximate the nonlinear model through a linear interpolation scheme. ${ }^{31}$

\section{B. $N_{L}$ Speed Controller}

A block diagram of the set point controller is shown in Fig. 8, where the primary control loop is closed on $N_{L}$. The gains $K_{P}$ and $K_{I}$ of the PI controller are scheduled based on altitude, Mach number, and the PLA. ${ }^{33}$ This aids in producing a critically damped response at different power levels. The integrator term contains an integral anti-wind-up protection scheme ${ }^{34}$ that includes the gain (IFB) as shown in Fig. 8. The output of the controller, $W_{f}$ Reg, is the controller's desired fuel flow rate and $W_{f}$ Cmd is the actual fuel flow rate after the $\mathrm{min} /$ max protection logic at the last time step. For this study, the controller was designed to achieve the typical bandwidth of the fuel pump actuator of $6 \mathrm{~Hz}$ with a constraint of producing a gain margin of at least $6 \mathrm{~dB}$ and a phase margin greater than 60 degrees. This was achieved while maintaining a critically damped closed-loop response.

\section{Protection Logic Limiter}

For safety and operability, protection logic is used to regulate excessive transients and other operating limits that could lead to stall or other engine failures, as shown in Fig. 7. This is done by monitoring sensed parameters for the engine shafts' acceleration, combustor pressure, maximum $N_{L}$ and high pressure shaft speed $\left(N_{H}\right)$ speeds, and the ratio unit (RU), which is $W_{f}$ over compressor discharge static pressure (Ps3). 


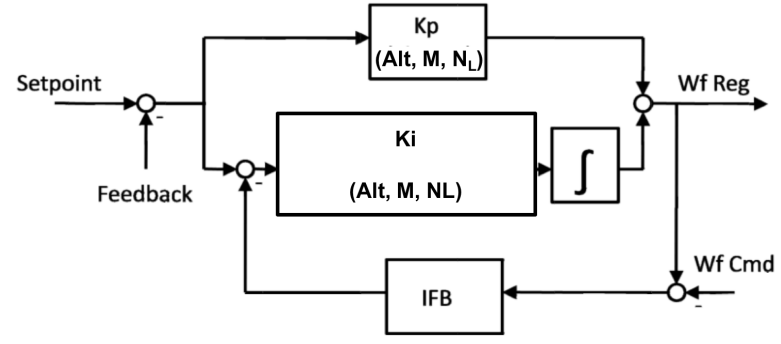

Figure 8: Gain scheduled set point controller block diagram.

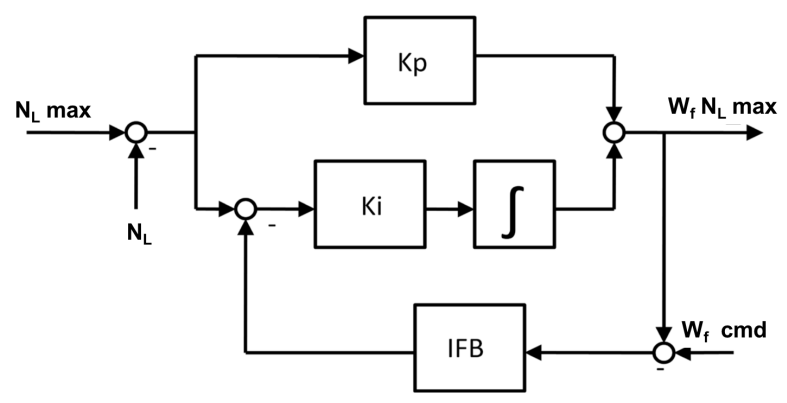

Figure 9: Diagram of $N_{L}$ maximum speed limiter.

The acceleration of the engine shafts' serves as a proxy for stall margin (SM) since there is no on-board sensor for SM. The final $W_{f}$ signal that is provided to the fuel metering valve is the outcome of a selection process using a min/max approach. The controller architecture developed here is based on a modern controller architecture for a commercial turbofan engine. ${ }^{35}$

The protection logic is designed to ensure that critical variables do not exceed their limits. Each limit regulator is implemented as a PI controller, similar to the set point controller shown in Fig. 8. The main difference here is that the PI gains are constant, not scheduled. The primary limiter is the maximum $N_{L}$ speed limiter, as this will be the primary safety limiter for the DART facility. The purpose of this limiter is to prevent an over speed condition that can cause conditions for excessive fatigue in the rotating components. A diagram of the maximum $N_{L}$ limiter structure is shown in Fig. 9 and is very similar to the general structure of all the other limiters used in this study. The $\max N_{L}$ speed is a constant value, as are the controller gains $(K p, K i$, and $I F B)$. The output of the $\max N_{L}$ speed controller is $W_{f}$.

\section{Results}

This section contains the comparison of the T-MATS nonlinear engine model against the data obtained from the DGEN 380 hardware ground test and the WESTT test bench at cruise. Once the nonlinear model fidelity is established, the PWLM control model is compared to the nonlinear model; the controller is designed using the PWLM and presented to illustrate meeting the control margin objectives. Finally, the closed loop response of the nonlinear engine model is presented.

\section{A. Nonlinear Model Comparisons}

An initial ground test of the DGEN 380 engine was conducted inside the AAPL prior to the completion of the DART facility. Data from this test along with data from the WESTT simulation test bench is used for comparison of the T-MATS nonlinear model at ground level. To assess the nonlinear model at cruise data is used from across various power settings using the WESTT test bench.

\section{DGEN 380 Engine Ground Test}

An initial ground test of the DGEN 380 was conducted using a stair case transient step in the PLA starting from a flight idle condition to full power. At the completion of the stair case steps a large deceleration transient is conducted followed by a large acceleration transient. The engine data acquired during this test was limited to that being used by the Full Authority Digital Engine Control (FADEC). It should be noted that the data presented was obtained during the initial check out test in the AAPL prior to the development of the DART facility and the simulation comparison are open loop or without a controller. The rotational shaft speeds are shown in Fig. 10, where the data is normalized by the maximum value of the corresponding engine test data. For this test, the DGEN 380 is running a closed loop $N_{L}$ fuel flow controller; however, for comparisons the fuel flow signal from the engine test is directly provided to the nonlinear T-MATS model running open loop. In Fig. 10 (a) the normalized $N_{L}$ speed is shown in the top plot and the bottom plot shows the percent error from the test data to simulation data. The blue solid line is the nonlinear T-MATS model, the black dotted line is the engine test data, and the red dashed line is the WESTT test bench data. 

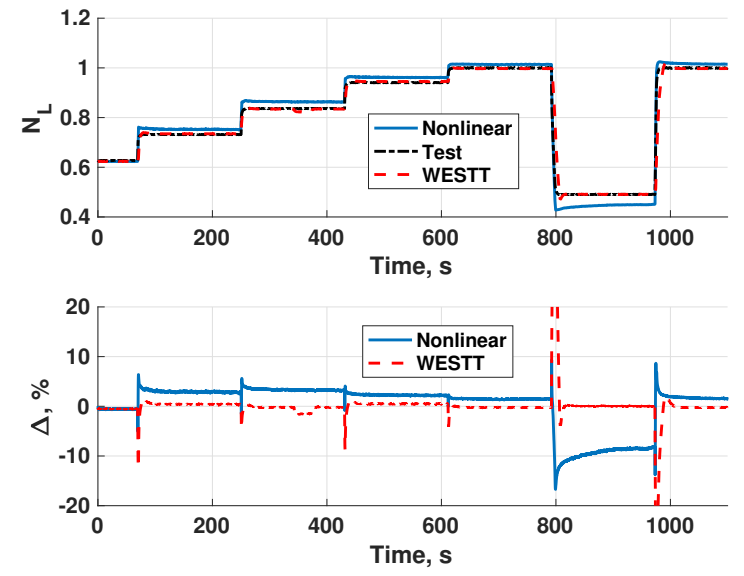

(a) $N_{L}$ Shaft Speed
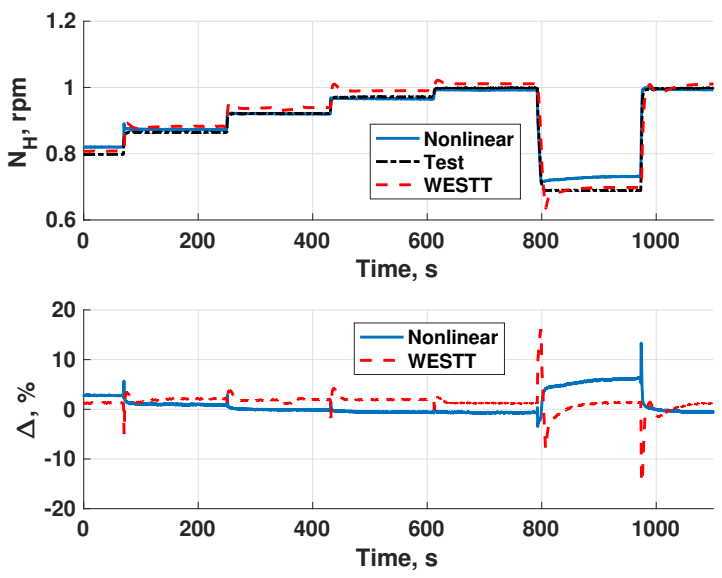

(b) $N_{H}$ Shaft Speed

Figure 10: Transient response of the nonlinear T-MATS DGEN model compared to DGEN 380 engine test data for rotational shaft speeds.

The $N_{L}$ response shown in Fig. 10 (a) is accurate during the smaller stair case transient maneuvers to within a few percent. During the larger transient maneuvers of the engine the error grows, but is still in general less than $10 \%$. The largest error is during the deceleration, while the large acceleration transient tracks well with the test data. The $N_{H}$ response shown in Fig. 10 (b) has very similar trends to the response of $N_{L}$. The $N_{H}$ response has less variation with the test data when compared to $N_{L}$. Also, $N_{L}$ has the largest difference with test data during the large deceleration transient. The WESTT test bench and nonlinear engine model provide comparable modeling accuracy during the steady state and transient portion of the engine test. Ps3 is another critical control parameter for the engine and comparison results are shown in Fig. 11. Here, it can be seen that the nonlinear T-MATS model has a steady state offset from the engine sensed parameter. The WESTT test bench model has a lower steady state error; however, the nonlinear model has a lower error during transients. This is particularly noticeable during the large deceleration transient, where the WESTT model initially tracks to a pressure drop and then experiences a delay causing a larger deviation from the engine test data. The accuracy of the nonlinear model is sufficient to be used for initial control design.
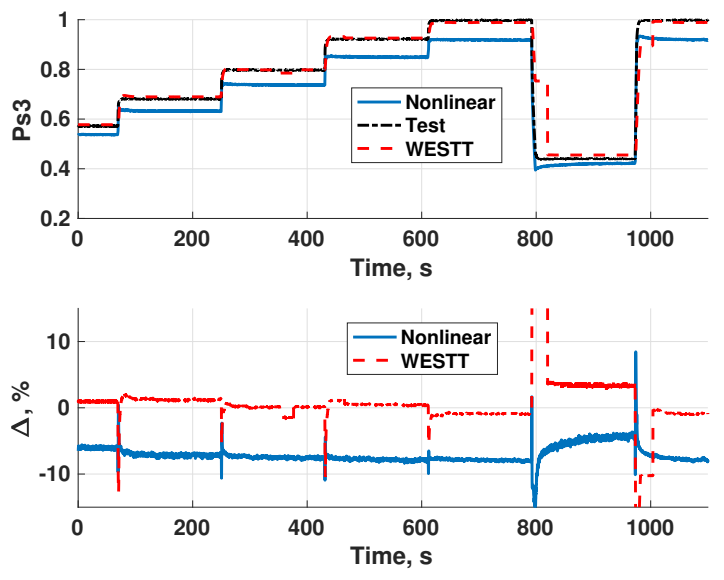

Figure 11: Transient response of the nonlinear T-MATS DGEN model compared to DGEN 380 engine test data for Ps3. 


\section{WESTT Simulation Full Flight Envelope}

In the previous subsection, it was shown that the WESTT test bench LPV model is accurate to within $10 \%$ of engine test data outside of the large transients. While it would be ideal to have engine test data at altitude, the WESTT test bench provides a reasonable check on the fidelity of the nonlinear T-MATS model beyond ground testing. This is particularly true for the cruise condition, where the transient maneuvers will be significantly less than those during ground takeoff where the WESTT test bench experiences its greatest errors. For the results presented, the closed loop WESTT test bench model is run at a cruise condition of $10,000 \mathrm{ft}$ and Mach 0.33. The output fuel flow signal is used to set the fuel flow for the open-loop nonlinear T-MATS engine model. The models are simulated at eight different fuel values to determine the fidelity of the nonlinear model by comparing three metrics. First is the fan pressure ratio (FPR), which is the pressure ratio across the fan component, fan exit pressure (P21) divided by inlet pressure (P2). Second, the overall pressure ratio (OPR) that is the pressure ratio across the compressive components, HPC exit pressure (P3) divided by P2. Third, the high pressure turbine inlet temperature (T4). Each of these metrics is shown as a function of $N_{L}$, scaled by the maximum speed setting in Fig. 12 (a), where the blue solid line is the nonlinear T-MATS model and the red dashed line is the WESTT test bench data. The percent difference between the T-MATS model and the WESTT test bench data is shown in Fig. 12 (b) to be within approximately 5\% across the power setting range. A general trend shown in Fig. 12 is that the models agree best at the higher power settings (farthest to the right), with a greater difference at lower power.
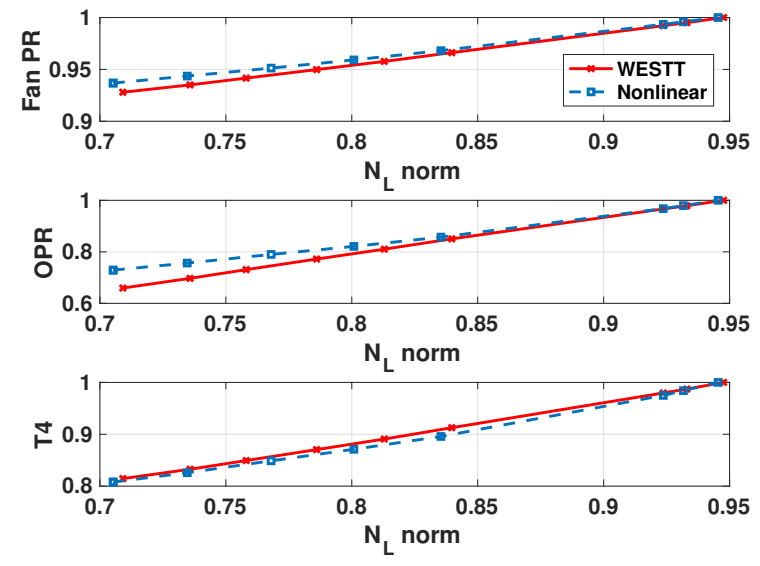

(a) Steady State
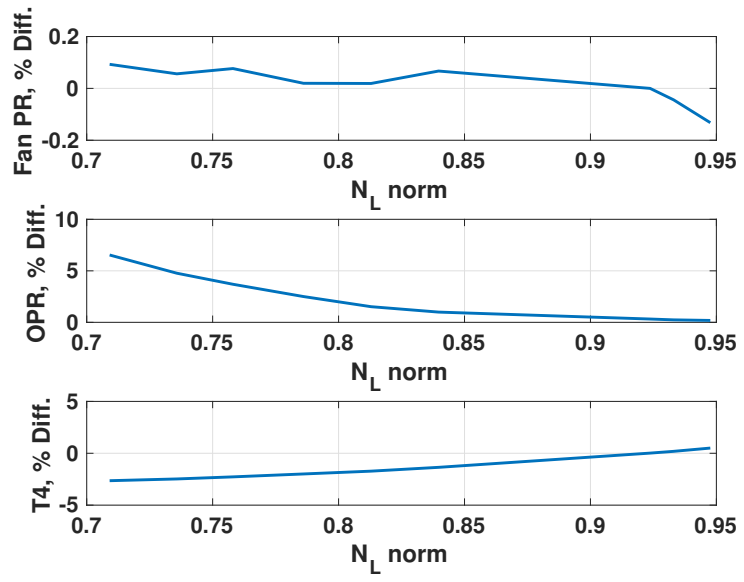

(b) Precent Difference

Figure 12: Steady-state comparison of the nonlinear T-MATS DGEN model to the Price Induction WESTT virtual engine test bench for fan pressure ratio (FPR), overall pressure ratio (OPR), and $\mathrm{T} 4$ as a function of $N_{L}$ speed shaft at cruise.

The comparison of the nonlinear T-MATS model to available test data at ground condition and simulation data at cruise shows a good matching to generally within $10 \%$. While the model was developed with limited available data and lacking specific performance maps, the accuracy obtained is suitable for initial controller development. Once the DART facility is fully operational, more engine test data can be obtained for a rescaling of the current generic performance maps used in the nonlinear model, which will improve accuracy.

\section{B. Nonlinear Model Comparisons to Piece Wise Linear Model}

An accurate linear model is required for the baseline closed loop controller design for the nonlinear TMATS model. To account for the full flight envelope a PWLM model is created at 336 distinct operating conditions across altitude, Mach number, and $N_{L}$ speed. To ensure that the linear model accurately reflects the nonlinear model, a steady state and transient comparison of key control parameters of interest $\left(N_{L}\right.$, $\left.N_{H}, P s 3, E G T, F_{n}\right)$ are shown in Fig. 13. For the steady-state results shown in Fig. 13 (a), 1,000 random operating conditions are simulated for both the nonlinear and PWLM models spanning the full operating envelope. The blue line shows the steady state percent difference, and the red dashed line shows the mean 
percent difference across all 1,000 operating points. The steady state results shown in Fig. 13 (a) illustrate that for most of the parameters of interest the mean error is less than $0.5 \%$. The only outlier is the net thrust, with a mean error of less than 1.5\%. The transient comparison is shown in Fig. 13 (b), where 1,000 operating conditions are simulated with a $20 \%$ fuel flow step change. It should be noted that if the random condition is near full power, the step change is limited to the maximum power of the engine. Transient comparison data are obtained 0.1 seconds prior to the step change until the response settles to within one percent of its final value. The transient response for the same parameters of interest from the steady state results are shown in Fig. 13 (b), where the dashed red is the mean percent difference and blue is the maximum percent difference. The mean for the transient results are across time for a single operating point simulation, instead of across the final steady value across all operating points in the steady state plot. General trends of the mean percent difference between the steady and transient simulations are similar to within a tenth of a percent. For most of the parameters of interest, the maximum percent difference shown in Fig. 13 (b) is less than $2 \%$, where the largest errors are again in the net thrust.
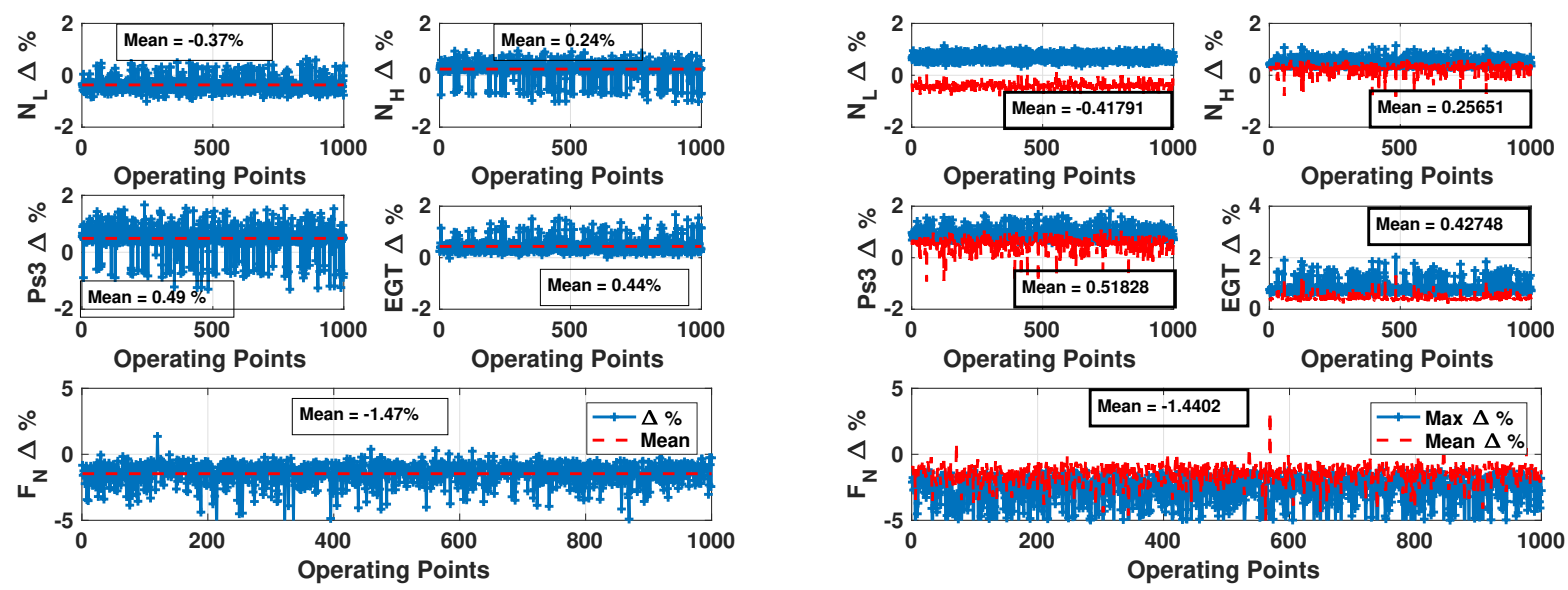

(a) Steady State

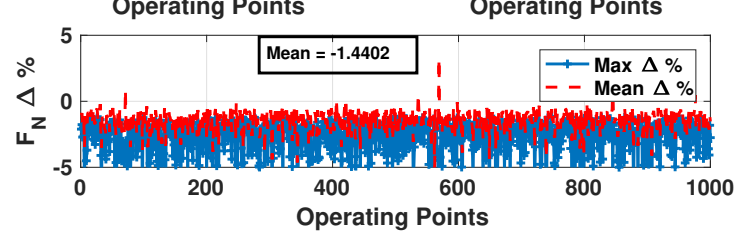

(b) Transient

Figure 13: Nonlinear engine model comparisons to PWLM models across 1,000 random operating conditions, the transient comparisons have a $20 \%$ fuel flow step change.

From the results last shown, the accuracy obtained for the PWLM was considered sufficient for initial control design that follows. If there is a need for greater accuracy, the PWLM can be generated at a larger number of operating conditions to reduce the nonlinear effects between linear operating points.

\section{Controller Design}

An engine controller architecture using $N_{L}$ feedback to modulate the fuel flow that includes limit logic for over speeds, acceleration, and maximum temperatures is developed using the PWLM. The closed loop controller uses a simple PI gain approach, where the goal of the controller design is to obtain $K_{I}$ and $K_{P}$ gains that provide the desired gain margin of $6 \mathrm{~dB}$ and phase margin of 60 degrees for the closed loop system. For the main fuel flow controller, the actuator dynamics are included in the design, but sensor noise is initially neglected. At each operating point in the flight envelope that a state space system exists for the PWLM an optimization is conducted to obtain the controller gains ${ }^{36}$ as shown in Fig. 14. The margins are shown as a three dimensional color coded plot to illustrate changes in the margin across the flight envelope, where red indicates less margin and blue indicates more margin. It is shown in Fig. 14 (a) that the desired $6 \mathrm{~dB}$ is obtained across the full fight envelope, the lower value of the margin can be seen to tend towards high $N_{L}$ speed values. This implies that at lower power settings the fuel flow controller has more gain margin. The phase margin is shown in Fig. 14 (b), where the trend for the gain margin does not seem to hold. For the controller designed in this study there is less variation in the phase margin across various operating condition. In addition, the phase margin obtained exceeds the goal of maintaining at least 60 degrees.

The additional phase margin in the controller could be traded to increase the responsiveness of the controlled parameter. However, there should be enough additional margin to account for PWLM inaccuracies, 


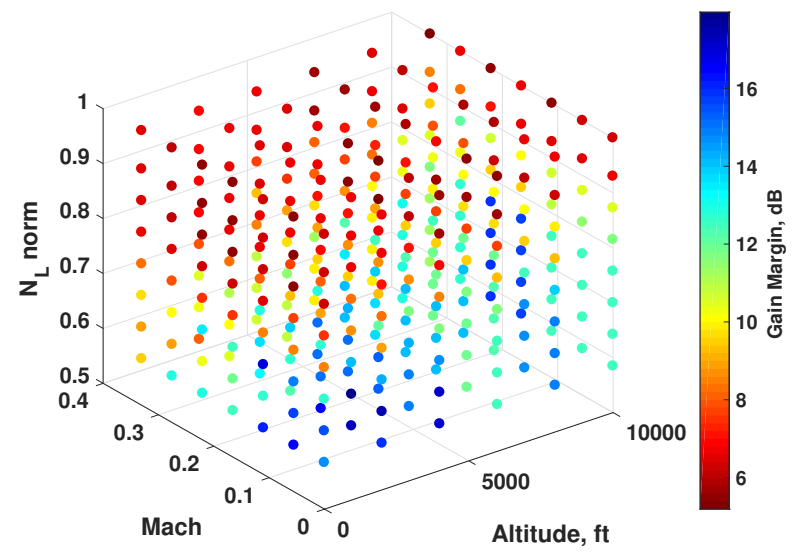

(a) Gain Margin

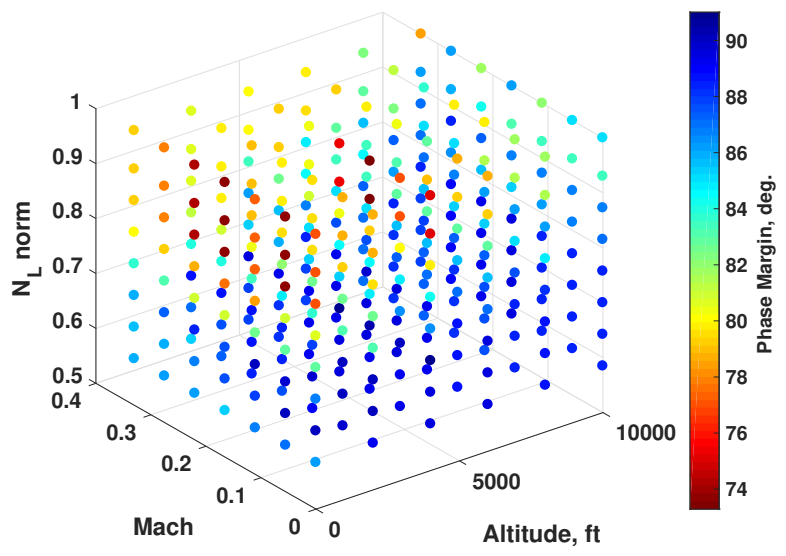

(b) Phase Margin

Figure 14: Controller gain and phase margin as applied to the PWLM with fuel valve actuator dynamics.

nonlinearities of the model or system the controller will be applied to, sensor noise, and disturbances. The transient requirements of the turbofan engine are defined by a Federal Aviation Administration (FAA) transient takeoff requirement. ${ }^{37}$ This is defined as going from flight idle to $95 \%$ power within five seconds. Engine settling time for the closed loop PWLM engine response to a step change is shown in Fig. 15 with the same general three dimensional plot format. A worst case settling time for the PWLM is shown to be approximately 3.2 seconds, well within the FAA requirement. The general settling time trend shown in Fig. 15 illustrates that at conditions closer to flight idle, such as low altitude, low Mach, and low $N_{L}$ speed the settling time is longer. This makes intuitive sense, given the lower starting speed of the engine shafts it will take longer to overcome inertia to speed up the turbofan engine.

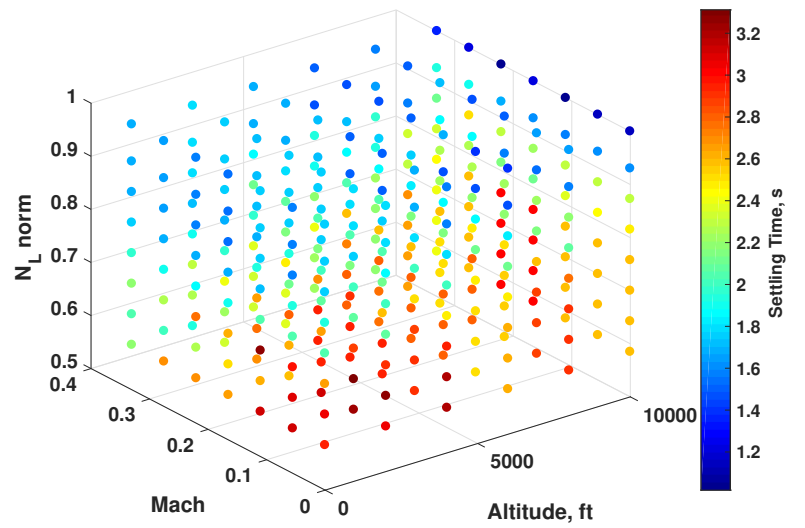

Figure 15: Closed loop control $N_{L}$ speed settling time to step change in the PWLM control model across 336 flight operating conditions.

\section{Closed Loop Dynamic Response}

The controller gains obtained from the design process on the PWLM are now applied to the nonlinear TMATS model to investigate the closed loop response of the baseline DGEN 380 model. This model contains sensor noise and a PLA to $N_{L}$ set point function neglected in the PWLM. To illustrate the performance of the limit logic, the engagement of the max $N_{L}$ limiter is shown in Fig. 16 due to a large fuel flow transient at 
five seconds. The top plot shows the $N_{L}$ response with the limiter (solid blue line) and without the limiter (dashed teal line). A dashed red line indicates the limiter threshold, all values in the plot are normalized by the $N_{L, \max }$ value. The top plot of Fig. 16 shows the control limiter was able to protect the engine by preventing the $N_{L}$ response from exceeding the over speed value the $N_{L}$ set point is commanding the engine to follow. The bottom plot shows when the limiter is engaged. The integral windup protection produces a smooth transition during the switch between the main fuel flow controller and max $N_{L}$ limit controller as observed at 5.8 and 9.9 seconds.
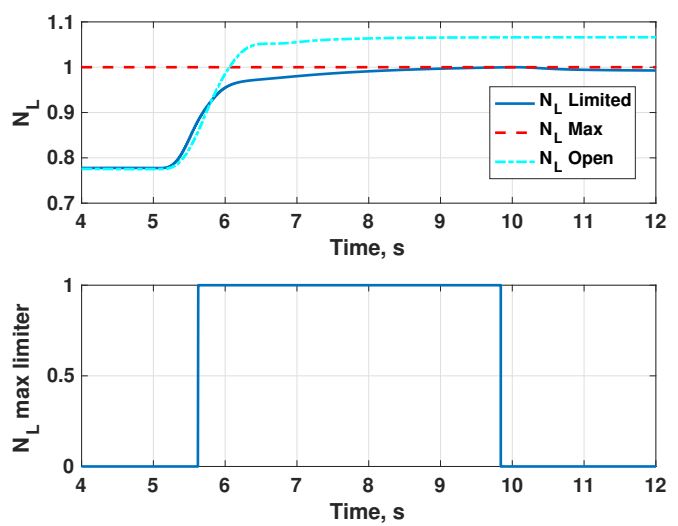

Figure 16: Engine control response to a large fuel flow change that engages the $N_{L}$ max speed limiter, The top plot shows the $N_{L}$ response with and without the $N_{f, \max }$ limiter engaged and limit threshold. response with and without the limiter. The bottom plot shows when the limiter is engaged.
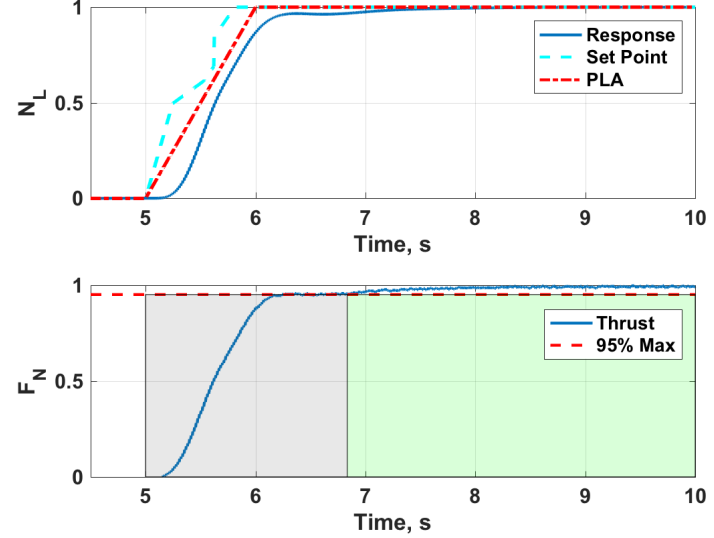

Figure 17: FAA turbofan engine transient requirement for going from flight idle to $95 \%$ power within five seconds.

To ensure that the $N_{L}$ baseline controller as applied to the nonlinear T-MATS model meets the FAA requirement ${ }^{37}$ threshold, a PLA command at the five second mark from flight idle to maximum power is shown in the top plot of Fig. 17. All of the values shown are normalized such that zero represents a flight idle condition and one is at maximum power. The top plot shows the resulting $N_{L}$ commanded set point and $N_{L}$ response from the nonlinear model. The $N_{L}$ speed is correlated with the overall net thrust of the engine shown in the bottom plot of Fig. 17. The dashed red line is the $95 \%$ maximum power threshold that the FAA requires to be met within five seconds. The grey shaded portion of the plot indicates the duration from the start of the transient to meeting the objective of the requirement and the green shaded portion of the plot shows the additional time available to meet the requirement. Here the closed loop response of the engine was able to meet the requirement within two seconds from the initial start of the transient. The initial controller design for the DGEN 380 meets the desired five second transient response applied to the nonlinear model.

\section{Conclusions}

A nonlinear dynamic model and propulsion controller is developed for a small-scale turbofan engine. The small-scale turbofan engine is modeled on the Price Induction company's DGEN 380. This paper contains information regarding the DGEN 380 engine and their test bench simulator, the WESTT CS/BV, which represents the DGEN 380 and includes an engine control unit. Comparisons of the nonlinear dynamic turbofan engine model to engine test data and the WESTT simulation are provided. During engine transients, the nonlinear model typically agrees within $10 \%$ error, even though the nonlinear model was developed with limited available engine data. A gain scheduled proportional integral low pressure shaft speed $\left(N_{L}\right)$ controller is developed with limiter safety logic to reflect the baseline DGEN 380 controller. The controller provides desired gain and phase margins and is tested to meet Federal Aviation Administration transient propulsion system requirements. There is a need for demonstrating advanced control architectures and technologies using real-time systems and hardware to move beyond showing benefits only in simulation. The small scale 
DGEN 380 provides a cost effective means to accomplish advanced controls testing on a relevant turbofan engine platform. The propulsion controller developed here can provide a baseline from which future advanced controller development can be compared. NASA has acquired a DGEN 380 engine and installed it in the DGEN Aero-propulsion Research Turbofan (DART) facility. Further improvements to the baseline model to enable better matching of key control parameters to the installed engine of the DART facility will be achieved as more engine test data becomes available.

\section{Acknowledgements}

The authors would like to thank the Transformational Tools and Technologies Project under the NASA Aeronautics Mission Directorate's Transformative Aeronautics Concepts Program for their support of this work, and insights from Price Induction's Benjamin Gomer on the operation of the DGEN 380 turbofan engine. In addition, the authors would like to express their appreciation for the efforts of the test-facilities operations, maintenance and engineering staff at the Aero-Acoustic Propulsion Laboratory.

\section{References}

${ }^{1}$ Litt, J. S., Simon, D. L., Garg, S., Guo, T.-H., Mercer, C., Millar, R., Behbahani, A., Bajwa, A., and Jensen, D. T., "A Survey of Intelligent Control and Health Management Technologies for Aircraft Propulsion Systems," Journal of Aerospace Computing, Information, and Communication, Vol. 1, 2004.

${ }^{2}$ Liu, Y., Dhingra, M., and Prasad, J. V. R., "Benefits of Active Compressor Stability Management on Trubofan Engine Operability," Journal of Engineering for Gas Turbines and Power, Vol. 131, 2009.

${ }^{3}$ DeCastro, J. A., "Rate-Based Model Predictive Control of Turbofan Engine Clearance," Journal of Propulsion and Power, Vol. 23, No. 4, July-August 2007.

${ }^{4}$ Henriksson, M., Gronstedt, T., and Breitholtz, C., "Model-Based On-Board Turbofan Thrust Estimation," Control Engineering Practice, Vol. 19, March 2011, pp. 602-610.

${ }^{5}$ Shi, Y. and Zhao, J., "Switching Control for Aero-Engines Based on Switched Equilibrium Manifold Expansion Model," IEEE Transactions on Industrial Electronics, Vol. 64, No. 4, April 2017.

${ }^{6}$ Qin, J., Huang, J., and Pan, M., "An Optimal Augmented Monotonic Tracking Controller for Aircraft Engines with Output Constraints," Energies, Vol. 10, No. 1, 2017.

${ }^{7}$ Xiaofeng, L., Jing, S., Yiwen, Q., and Ye, Y., "Design for Aircraft Engine Multi-Objective Controllers with Switching Characteristics," Chinese Journal of Aeronautics, Vol. 27, No. 5, 2014, pp. 1097-1110.

${ }^{8}$ Yu, B., Shen, E., Huang, Y., and Lu, F., "Research on Self-Learning Control Method for Aircraft Engine Above Idle State," Advances in Mechanical Engineering, Vol. 8, No. 6, 2016, pp. 1-10.

${ }^{9}$ Wang, X., Zhao, J., and Sun, X.-M., "Overshoot-free Acceleration of Aero-engines: An Energy-based Switching Control Method," Control Engineering Practice, Vol. 47, February 2016, pp. 28-36.

${ }^{10}$ Connolly, J. W., Csank, J., and Chicatelli, A., "Advanced Control Considerations for Turbofan Engine Design," AIAA 2016-4653, July 2016.

${ }^{11}$ Csank, J. and Connolly, J. W., "Model-Based Engine Control Architecture with an Extended Kalman Filter," AIAA 2016-1623, 2016

${ }^{12}$ Connolly, J., Csank, J., Chicatelli, A., and Kilver, J., "Model-Based Control of a Nonlinear Aircraft Engine Simulation using an Optimal Tuner Kalman Filter Approach," AIAA 2013-4002, 2013.

${ }^{13}$ Wang, T., Jobredeaux, R., Pakmehr, M., Vivies, M., and Feron, E., "An Application of a Prototype Credible Autocoding and Verification Tool-Chain," IEEE 6979438, October 2014.

${ }^{14}$ Dufour, G., Rosa, N. G., and Duplaa, S., "Validation and Flow Structure Analysis in a Turbofan Stage at Windmill," Journal of Power and energy, Vol. 229, No. 6, 2015, pp. 571-583.

${ }^{15} \mathrm{Pi}$, J., Fu, J., Jiang, S., and Kong, Q. G., "Properties of the Small-Size Turbofan Engine Burning Biofuels," Biofuels, 2016.

${ }^{16}$ Culmone, M. V., Rosa, N. G., and Carbonneau, X., "Sensitivity Analysis and Experimental Validation of Transient Performance Predictions for a Short-Range Turbofan," GT2016-57257, June 2016.

17 "Price Induction DGEN Engine Specifications," http://www.price-induction.com/dgen-engine/, December 2015.

${ }^{18}$ Berton, J. J., "System Noise Prediction of the DGEN 380 Turbofan Engine," Journal of Aircraft, Vol. 53, No. 6, 2015, pp. $1779-1786$.

${ }^{19}$ Sutliff, D. L., Brown, C. A., Bayon, B., and Sree, D., "Farfield Acoustic Characteristics of the DGEN 380 Turbofan Engine as Measured in the NASA Glenn Aero-Acoustic Propulsion Laboratory," AIAA 2016-3006, May 2016.

${ }^{20}$ Price Induction, "WESTT CS/BV User Manual," MAU-000002-B03, 2012.

${ }^{21}$ Chapman, J. T., Lavelle, T. M., May, R. D., Litt, J. S., and Guo, T., "Propulsion System Simulation Using the Toolbox for the Modeling and Analysis of Thermodynamic Systems (T-MATS)," 2014-218410, November 2014.

${ }^{22}$ Cooper, B. A., "A Large Hemi-Anechoic Chamber Enclosure for Community-Compatible Aeroacoustic Testing of Aircraft Propulsion Systems," Journal of the Institute of Noise Control Engineering of the USA, January 1994.

${ }^{23}$ Culley, D., Thomas, R., and Saus, J., "Integrated Tools for Future Distributed Engine Control Technologies," GT201395118, June 2013.

${ }^{24}$ Spang, H. A. and Brown, H., "Control of Jet Engines," Control Engineering Practice, Vol. 7, No. 9, 1999, pp. $1043-1059$. 
${ }^{25}$ Chen, C., Sui, Y., and j. Zhao, "Coordinated Switching Control of Thrust Tracking and Safety Protection for AeroEngines," IEEE 2014-6895616, 2014.

${ }^{26} \mathrm{Du}, \mathrm{X}$. , Richter, H., and Guo, Y. Q., "Multivariable Sliding-Mode Strategy with Output Constraints for Aeroengine Propulsion Control," Journal of Guidance, Control, and Dynamics, Vol. 39, No. 7, 2016, pp. 1631-1642.

${ }^{27}$ Xiaofeng, L., Shi, J., Yuan, Y., and An, S., "Multiobjective Control System Controllers Design Based on Switching and Applications," IEEE Aerospace and Electronic Systems Magazine, Vol. 30, No. 1, February 2015, pp. 32-42.

${ }^{28}$ Zheng, Q. and Zhang, H., "A Global Optimization Control for Turbofan Engine Acceleration Schedule Design," Proceedings of the Institution of Mechanical Engineers: Journal of Aerospace Engineering, December 2016.

${ }^{29}$ Liu, X. and An, S., "Smooth Switching Controller Design for Multiobjective Control Systems and Applications," Journal of Aerospace Engineering, Vol. 29, No. 4, July 2016.

${ }^{30}$ Pakmehr, M., Fritzgerald, N., Feron, E. M., Shamma, J. S., and Behbahani, A., "Gain Scheduling Control of Gas Turbine Engines: Stability by Computing a Single Quadratic Lyapunov Function," GT2013-96012, June 2013.

${ }^{31}$ Lawrence, D. A. and Rugh, W. J., "Gain Scheduling Dynamic Linear Controllers for a Nonlinear Plant," Automatica, Vol. 31, No. 3, 1995, pp. 381-390.

${ }^{32}$ Rugh, W. J., "Analytical Framework for Gain Scheduling," IEEE Control Systems Magazine, Vol. 11, 1991, pp. 74-84.

${ }^{33}$ Csank, J., Ryan, M., Litt, J. S., and Guo, T., "Control Design for a Generic Commercial Aircraft Engine," NASA/TM 2010-216811, 2010.

${ }^{34}$ Martin, S., Wllace, I., and Bates, D. G., "Development and Validation of a Civil Aircraft Engine Simulation Model for Advanced Controller Design," Journal of Engineering for Gas Turbines and Power, Vol. 130, No. 5, June 2008.

${ }^{35}$ May, R., Csank, J., Litt, J. S., and Guo, T., "Commercial Modular Aero-Propulsion System Simulation 40K," NASA/TM 2010-216810, 2009.

${ }^{36}$ Astrom, K. J. and Hagglund, T., Advanced PID Control, Instrumentation, Systems, and Auomation Society, 2006.

${ }^{37}$ FAA, "Federal Aviation Regulatoin (FAR): Title 14 Part 33.73 Power or Thrust Response," 2013. 